\title{
Multi-Criteria Decision-based Path Planning for Data Collection in Fuzzy-Cluster based Large Sensor Networks
}

\author{
*Sunil Kumar Singh • Prabhat Kumar
}

Received: date / Accepted: date

\begin{abstract}
Wireless sensor networks (WSN) are widely used to gather information using wireless communication, but due to the confined power of sensor nodes, it is a predominant task to make WSN energy-efficient. Generally, a sensor node finds routes towards the base station (BS) to transmit the data. The sensor node transmits information at once or via neighbor nodes in a multi-hop manner. The nodes close to the BS transmit more data than other nodes. The nodes close to the BS tend to deplete their power quicker than different nodes in the network. This issue is known as the hotspot problem, leading to network separation and reducing the lifetime of the sensor network. The mobile sink as a better strategy to dispose of the hotspot problem in the course of information transmission, but the most critical challenges are finding sojourn points and path planning. This paper makes use of a cluster head $(\mathrm{CH})$ as a sojourn point and multi-criteria decision strategy for path planning by the mobile sink at some stage in information collection. This paper contributes a fuzzybased clustering algorithm that uses fuzzy logic to decide on cluster heads (CHs). The TOPSIS (Technique for Order Preference by Similarity to Ideal Solution) approach used to figure out the mobile sink's route. Based on this route, the mobile sink will move to collect data from every $\mathrm{CH}$ in the network. This scheme receives an order of $\mathrm{CHs}$ to visit and collect the data with the mobile sink. The simulation outcomes exhibit
\end{abstract}

*School of Computer Science and Engineering,

VIT-AP University, Amaravati, A.P.

Department of Computer Science and Engineering,

National Institute of Technology Patna,

Bihar, India.

E-mail: sunil.singh@vitap.ac.in, prabhat@nitp.ac.in

*Mobile-+91-9155238352

*ORCID iD- https://orcid.org/0000-0001-5262-6636 that this strategy performs better than the other protocols, which uses multi-hop information transmission for extending the lifetime of wireless sensor networks and one parameter for route planning.

Keywords Wireless Sensor Network, Cluster head (CH), Mobile sink, TOPSIS, Path planning, Hotspot

\section{Introduction}

A WSN is a network where a large number of small sensor nodes are densely deployed, and it works in cooperation for collecting data and communicating with the base station (BS) Akyildiz et al (2002), Fadel et al (2015). In most of the WSN, hundreds to thousands or even more than that sensor node (also called mote) are present. WSNs are widely used in defense surveillance, vehicle tracking, environment, health monitoring, disaster relief, etc. The sensor node is a significant component of any WSN. A sensor node is composed of several hardware parts: transducer with an antenna, a microcontroller for computation, sensing unit with A/D converter to sense, and converting the analog signal into digital, a memory unit for storage and a battery as a source of energy. Since a sensor node consists of several resources but in restricted mode, these are directly or indirectly related to the performance of the WSN. Therefore, the proper utilization of these resources is a must during any routing or data collection. Among those resources, energy is vital because its fast drainage directly affects the performance of the whole network. Therefore, saving battery life is the top priority in our work.

Battery life can be improved by minimizing the number of communications in the network, so an efficient routing protocol and duty cycle is required Debasis 
et al (2020). Generally, two types of routing are used in WSN: single-hop and multi-hop based on transmission from the sensor node to the BS Goyal and Tripathy (2012). Clustering supports both types of routing effectively, but multi-hop is mostly preferred in large area networks Singh et al (2017b). In clustering, optimal cluster formation and cluster head $(\mathrm{CH})$ selection are the major challenges that we have to resolve using the fuzzy controller to select $\mathrm{CH}$ and form a cluster Alrashidi et al (2020). In static BS and multi-hop routing, the hotspot is a severe issue that severely affects network performance. In a well-known hotspot [5], a sensor node near the BS consumes energy faster than other nodes because it transmits its data and other node data. The mobile sink has been considered a very effective way to reduce the hotspot problem in large sensor networks Sha et al (2017), Chauhan and Soni (2019). The path planning of the mobile sink is one of the significant challenges which requires a great effort to design an effective trajectory path for a mobile sink. Some path planning schemes have been developed based on the rendezvous point, but most of the proposed works have used simple predefined trajectory or single criteriabased path planning, which are not performed well in all the scenarios Singh and Kumar (2019), Pitke et al (2017). So, multi-criteria based path planning is necessary for the improvement of network performance Singh and Kumar (2020).

The aim of decision making based on multiple criteria is an important issue where system performance depends on the multiple parameters. In many situations, we can not make a decision based on the one-parameter if the multiple parameters are available. This is because we do not know the effect of all parameters on the performance. Multi-criteria decision making focuses on achieving preferred decisions from available alternatives using two or more criteria, which are often conflicting. The multi-criteria decision methods select among a distinct set of alternatives derived on multiple attributes and overall utility of the decision-makers. One of the famous and simple methods is TOPSIS which we have used in our model.

This paper contributes an efficient distributed clustering algorithm that uses fuzzy logic to elect CHs. The residual energy of the nodes and every node degree makes use of as the input variable for the fuzzy inference system (FIS) in this work to choose CH Rajaram and Kumaratharan (2020). The chance is the output parameter, which represents the probability of the nodes becoming NCH. The Mamdani method is used for fuzzy inference and centroid method for defuzzification. This scheme uses a mobile sink to eliminate the hotspot problem during data transmission.
The TOPSIS method is used to decide the route in which the mobile sink will move and collect data from each $\mathrm{CH}$ in the network. The decision criteria used for the TOPSIS method are the residual energy of the $\mathrm{CH}$, node degree of the $\mathrm{CH}$, and edge cost. The edge cost is calculated using the distance between the different nodes. A dynamic data collection path also helps in balancing the energy dissipation among sensor nodes.

The Remaining portion of the paper is organized in the following manner: Section 2 summarizes the related works. Section 3 elucidates the system model comprising with an energy model and explains the proposed protocol in detail. The simulation parameters with results and performance analysis are discussed in Section 4. Finally, Section 5 concludes the paper with future works.

\section{Related Works}

As our scheme uses clustering and route planning; therefore, right here at the beginning, primary clustering algorithms are discussed. After that, fuzzy-based unequal cluster and in last some famous mobile elements based schemes are explained.

The LEACH is the earliest clustering routing protocol based on distributed clustering Heinzelman et al (2000). This protocol works on rounds and at the beginning of each round, all nodes generate a random number between 0 and 1 . If the generated random numbers of the nodes are less than a threshold value, those nodes will be selected as $\mathrm{CH}$ for that round and broadcast a $\mathrm{CH}$ message to their member nodes. Though this protocol is energy-efficient, it has many drawbacks such as (i) nodes energy are not considered in $\mathrm{CH}$ selection, (ii) CHs transmit their data directly (single-hop) to the $\mathrm{BS}$, and (iii) $\mathrm{CHs}$ are placed anywhere in the clusters due to random selection. Further, several researchers have been contributed their idea to resolve these issues Singh et al (2017b), Heinzelman et al (2002), Qiang et al (2009), Biradar et al (2011) uses multi-hop path for communication to the BS. The multi-hop tree is created between the $\mathrm{CH}$ and the BS for communication. The multi-hop routing enhances the energy efficiency of the network, but it faces a severe problem named energy hole/hotspot, which we are trying to reduce in our scheme.

A balance cluster formation and selection of an optimal node as a $\mathrm{CH}$ are the major challenges of hierarchical routing. The fuzzy logic-based decision system can handle efficiently due to its partial truth nature. Bagci and Yazici have introduced the fuzzy logic-based unequal clustering to address the hotspot problem. The 
EAUCF Bagci and Yazici (2013) is a distributed clustering algorithm, where CHs are selected based on the transmission radius and distance from the BS. Initially, nominee CHs are made using a probabilistic model similar to LEACH. The residual energy and distance from the BS are used as input to the fuzzy inference system (FIS), which produces the competition radius for the cluster formation. The higher residual energy nodes among the tentative $\mathrm{CHs}$ are selected as the final $\mathrm{CHs}$. The normal nodes join their nearest $\mathrm{CH}$ and complete the cluster formation process. Further, they have extended their work with multi-objective fuzzy clustering algorithm (MOFCA) Sert et al (2015) which perform better for hotspot problem. Similarly, Baranidharan and Santhi have presented a distributed fuzzybased unequal clustering (DUCF) Baranidharan and Santhi (2016) with some different input parameters. Here, the fuzzy function creates different unequal clusters based on the distance from the BS to balance the energy consumption, which reduces the hotspot problem. A new version of fuzzy rule-based used with shuffled frog leaping algorithm (FSFLA) Fanian and Rafsanjani (2018) has proposed, which optimizes the inputs parameters based on the application. Randhawa and Jain developed a multi-objective load-balancing clustering (MLBC) Randhawa and Jain (2019) scheme with the help of multi-objective particle swarm optimization (MOPSO). Energy-Efficiency and Reliability are the two objective functions selected for the optimization.

Shah et al. Shah et al (2003) presented a three layers reference model where the lower layer consists of large numbers of sensor nodes that are sparsely deployed. In the middle layer, the mobile data carriers called MULES are visited randomly in the network and collects data from the nodes. These MULES transfer their collected data to the upper layer access point, which is IP enabled and connected with the rest of the world. This works like an opportunistic network that has more delays, and MULEs are uncontrolled. For large sensor networks, a data-collection protocol was presented using mobile sink by Zhao et al. This scheme focused on enhancing the lifetime of large area networks Zhao et al (2015). Several trees have been formed using multi-hop communication. The mobile sink walks in a fixed path and gathers data from the root node. A minimum spanning tree (MST) formed based on the minimum residual energy of nodes. The mobile sink travels to the MST vertices and takes data from these nodes. The main problem of this protocol is the energy hole occurs due to tree-based transmission. Singh et al. Singh et al (2016) proposed a protocol with the mobile base station. The whole rectangular area is divided into levels, and grids based on these levels and grids data are forwarded from the edge CHs. The mobile mule collected data from each level. There are two mules visited based on the round numbers, even or odd. This protocol is not appropriate for a vast area network due to centralized nature. The EDT and DAEDT Nitesh et al (2017) are the new techniques proposed by Kaswan for data collection using controlled mobility of the mobile sink. They have calculated rendezvous points with the help of residual energy. The network is flat and one-hop communication is the primary goal of this algorithm. An extension has been done in Singh et al (2016) with new trajectory patterns Singh et al (2018) of mobile mule. Authors have considered unequal clustering with a grid structure. Table 1 and 2 illustrate the comparative analysis of related clustering protocols and mobile sink based data collection protocols, respectively.

All the discussed protocols have two primary concerns optimal CH selection and path planning for data collection. In this work, we are trying to improve these with FIS based CH selection, and TOPSIS based ranking for $\mathrm{CHs}$ to collects data.

\section{Proposed Scheme}

\subsection{System and Energy Model}

In this work, we have considered Randomly deployed static sensor nodes along with a Mobile sink. All nodes are homogeneous and localized with the help of a localization algorithm discussed in Singh et al (2017a). The mobile sink has more energy, memory, communication range, and computation power as compare to simple sensor nodes. It moves with controlled mobility and uniform speed to collect data from the CHs. Continuously data is generated in the network.

In a sensor node, more than $90 \%$ of energy consumes in receiving and transmitting of data. Therefore, this work only examined the energy consumption during the reception and transmission of data. The proposed scheme has applied a first-order radio model. The transmitter amplifier uses both power loss model: free space $d^{2}$, and multi fading $d^{4}$. To transmit a $m$-bit data to a distance $d$ with the help of first-order radio model, this can be expended using Equations 1 and 2:

$$
E_{T x}(m, d)=m E_{e}+m \epsilon_{f s} d^{2}, \quad d<d_{0}
$$


Table 1: Comparative analysis of related clustering protocols

\begin{tabular}{|c|c|c|c|c|c|}
\hline Protocols & Year & Clustering & Overhead & Scalability & Energy efficiency \\
\hline LEACH Heinzelman et al (2000) & 2000 & Distributed & high & low & moderate \\
\hline $\begin{array}{l}\text { GBC Thanigaivelu and Murur- } \\
\text { gan (2012) }\end{array}$ & 2012 & Centralized & high & low & high \\
\hline $\begin{array}{l}\text { EEM-LEACH Antoo and Mo- } \\
\text { hammed }(2014)\end{array}$ & 2014 & Distributed & high & moderate & very high \\
\hline LPGCRA Liu et al (2010) & 2015 & Centralized & moderate & low & moderare \\
\hline $\begin{array}{llll}\text { GFTCRA Jannu } & \text { and Jana } \\
(2015) & & & \end{array}$ & 2016 & Centralized & moderate & moderate & high \\
\hline EEUC Chengfa Li et al (2005) & 2005 & Distributed & high & moderate & high \\
\hline PRODUCE Kim et al (2008) & 2008 & Semi-Centralized & low & moderate & moderate \\
\hline U-LEACH Kumar et al (2012) & 2012 & Distributed & low & high & high \\
\hline LUCA Lee et al (2011) & 2011 & Centralized & low & low & moderate \\
\hline EAUCF Bagci and Yazici (2013) & 2013 & Distributed & High & high & high \\
\hline $\begin{array}{l}\text { FBUC Logambigai and Kannan } \\
(2016)\end{array}$ & 2016 & Distributed & low & high & moderate \\
\hline $\begin{array}{l}\text { DFCR Nabajyoti and Hari } \\
(2018)\end{array}$ & 2018 & Distributed & moderate & high & high \\
\hline EEWC Pal et al (2020) & 2020 & Distributed & high & moderate & high \\
\hline
\end{tabular}

Table 2: Comparative analysis of related Mobile sinks based data collection protocols

\begin{tabular}{|c|c|c|c|c|}
\hline Protocols & Main contributions & Advantages & $\begin{array}{l}\text { Metrics } \\
\text { of } \\
\text { interest }\end{array}$ & $\begin{array}{l}\text { Network } \\
\text { Size }\end{array}$ \\
\hline $\begin{array}{l}\text { Juang et al } \\
(2002)\end{array}$ & $\begin{array}{l}\text { Attached sensor nodes as a Zebra collar on } \\
\text { Zebra to collect data }\end{array}$ & $\begin{array}{l}\text { to carry wild animals study } \\
\text { in a large area }\end{array}$ & Energy & $1000 K M^{2}$ \\
\hline $\begin{array}{l}\text { Shah et al } \\
(2003)\end{array}$ & $\begin{array}{l}\text { a three-tier architecture using mobile ele- } \\
\text { ments for data collection known as data } \\
\text { MULE }\end{array}$ & $\begin{array}{l}\text { Data collection in random } \\
\text { way from deployed area }\end{array}$ & Reliability & $\mathrm{N} / \mathrm{A}$ \\
\hline $\begin{array}{l}\text { Jain et al } \\
(2006)\end{array}$ & Mobile nodes work as a forwarding agents & $\begin{array}{l}\text { provide substantial savings } \\
\text { in energy }\end{array}$ & Energy & Large \\
\hline $\begin{array}{l}\text { Nesamony } \\
\text { et al (2007) }\end{array}$ & $\begin{array}{l}\text { formulate optimal path for mobile sink to } \\
\text { collect data }\end{array}$ & $\begin{array}{l}\text { Data collection from each } \\
\text { node }\end{array}$ & $\begin{array}{l}\text { Energy } \\
\text { \& Delay }\end{array}$ & large \\
\hline $\begin{array}{l}\text { Rao and } \\
\text { Biswas } \\
(2008)\end{array}$ & Optimize data collection using mobile sink & $\begin{array}{l}\text { a trade-off between energy- } \\
\text { efficiency and data delivery } \\
\text { delay }\end{array}$ & hotspot & $\mathrm{N} / \mathrm{A}$ \\
\hline $\begin{array}{l}\text { Xing et al } \\
(2008)\end{array}$ & $\begin{array}{l}\text { tree-based data collection scheme where } \\
\text { RPs are formed based on the label cover- } \\
\text { ing }\end{array}$ & $\begin{array}{l}\text { minimizes the overall path } \\
\text { length of the mobile elements }\end{array}$ & $\begin{array}{l}\text { Energy } \\
\text { \& Delay }\end{array}$ & $300 \mathrm{~m}^{2}$ \\
\hline $\begin{array}{l}\text { Singh et al } \\
(2016)\end{array}$ & $\begin{array}{l}\text { Grid-based clustering with a predefined } \\
\text { data mule }\end{array}$ & Simple path planning & Energy & $500 \mathrm{~m}^{2}$ \\
\hline $\begin{array}{l}\text { Kumar } \\
\text { et al }(2017)\end{array}$ & $\begin{array}{l}\text { a new data collection scheme based on la- } \\
\text { bel covering where some sub-set vertices se- } \\
\text { lected as the RPs from where mobile sink } \\
\text { collects data }\end{array}$ & Efficient data collection & Energy & $\begin{array}{l}.4 \mathrm{X} .6 \\
K M^{2}\end{array}$ \\
\hline $\begin{array}{l}\text { Singh et al } \\
(2018)\end{array}$ & $\begin{array}{l}\text { level and grid based unequal clustering with } \\
\text { two mobile sinks }\end{array}$ & $\begin{array}{l}\text { Simple and efficient data col- } \\
\text { lection scheme }\end{array}$ & Energy & $\begin{array}{l}.5 X .25 \\
K M^{2}\end{array}$ \\
\hline $\begin{array}{l}\text { Chang et al } \\
(2020)\end{array}$ & $\begin{array}{l}\text { adopted an appropriate transmission data } \\
\text { rate and collect data from each static sensor } \\
\text { nodes }\end{array}$ & reduces path length & Energy & $1 K M^{2}$ \\
\hline
\end{tabular}


$E_{T x}(m, d)=m E_{e}+m \epsilon_{m p} d^{4}, \quad d \geq d_{0}$

$\epsilon_{f s}$ and $\epsilon_{m p}$ are the required energies to enlarge the transmitted data in free space propagation and multipath fading respectively and $d_{0}$ is the threshold distance. The $E_{e}$ is the major power bump off to run the transmitter or receiver circuitry. In free space $f s$ propagation model, $d$ is constantly less than $d_{0}$ and in multipath fading $m p$ model $d$ is constantly higher than or equal to the $d_{0}$. The power consumption to receive an equal amount of data $\left(E_{R x}\right)$ can be computed by way of the usage of Equation 3.

$E_{R x}(m)=m E_{e}$

The proposed scheme contains three phases: (i) set-up, (ii) path planning, and (iii) data collection. The complete process of this scheme can be shown in Figure 1 with the help of a timeline.

\subsection{Set-up Phase}

In the beginning, after random deployment, every node $\left(N_{i}\right)$ finds its coordinates $\left(A_{i}, B_{i}\right)$ by way of using an efficient localization scheme Singh et al (2017a). Afterward, each node $N_{i}$ broadcasts a control packet with its details such as node ID, present-day strength and location coordinates. Each node maintains a neighbor table, which helps to calculate the node degree (total number of nodes in the range of a precise node) of that node. Suppose, a node $N_{i}$ receives a control packet from $N_{j}$, then the updated neighbour table of $N_{i}$ will be $\operatorname{Nbr}\left(N_{i}\right)=\left[\operatorname{Nbr}\left(N_{i}\right) \cup N_{j}\right]$. After that, all nodes will be aware of the neighbor's statistics like their distance, power, etc. This information will help in modeling the FIS for $\mathrm{CH}$ decision and cluster formation. The set-up section, more often than not, consists of $\mathrm{CH}$ selection and cluster formation. In this phase, clusters are shaped with FIS's help, and it is completely allotted in nature because of $\mathrm{CH}$ selection and cluster formation function based on local data only.

\subsubsection{CH Selection}

This scheme uses fuzzy logic for efficient $\mathrm{CH}$ selection. The fuzzy logic is an approach to handling any problem's uncertainties, which generally computes the degree of truth (membership function) instead of boolean logic (0 and 1). Several parameters are used to select the optimal $\mathrm{CH}$ in the clustering approach of the WSN, such as residual energy, node degree, node's distance from the BS, etc. Here, most of the parameters are

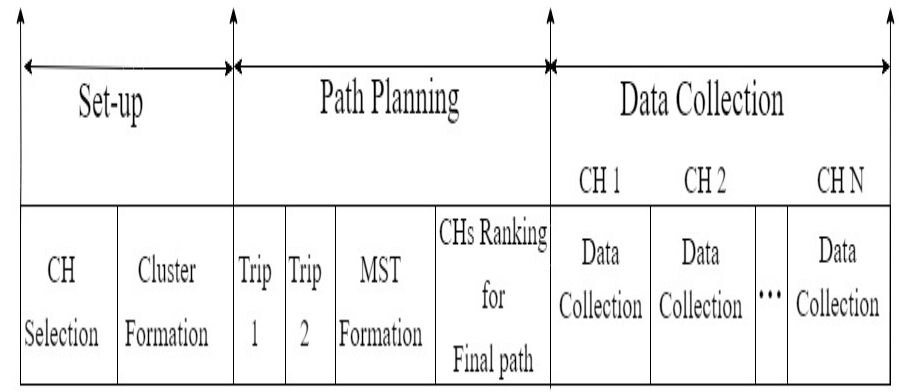

Fig. 1: A process time-line diagram of one round

variable and not fixed, so fuzzy logic is the best tool for selecting effective CHs. The FIS is a mapping tool in fuzzy logic that uses a set of inputs, and based on some predefined if-then rules; it shows outputs. The frequently used Mamdani inference approach is applied to compute the if-then rules. The different if-then rules for functional mapping with inputs and output are illustrated in Table 3. For defuzzification, the center of the area (COA) method is applied to calculate the chance value for $\mathrm{CH}$ selection. In order to select the $\mathrm{CH}$, two important parameters are used as inputs in the FIS system.

The residual energy is the first input parameter that is used in FIS for selecting the tentative $\mathrm{CH}$ as shown in Figure 2. This figure depicts the linguistic input variable for the residual energy, which is more, average and low. The trapezoidal membership function has been used for more and low linguistic variables, and the average triangular membership function has been used. The second input parameter is node degree, and for this parameter, the linguistic variables are sparse, fair, and dense. Figure 3 denotes the fuzzy input membership function distribution of node degree. A trapezoidal membership function represents the sparse and dense linguistic variable, and a triangular membership function denotes fair.

The only fuzzy output is chance variable, which selects the tentative CHs. Figure 4 illustrates the fuzzy set for fuzzy output variable to calculate a chance value. The nine linguistic outputs are very high, high, quite high, high average, average, low average, quite low, low and very low. The fuzzy if-then rules are shown in Figure 5. The FIS trapezoidal membership function and triangular membership function are mentioned in Equations 4 and 5 . 
Table 3: Fuzzy if-then rules for FIS

\begin{tabular}{|l|l|l|l|}
\hline $\begin{array}{l}\text { Rule } \\
\text { No. }\end{array}$ & $\begin{array}{l}\text { Residual } \\
\text { energy }\end{array}$ & $\begin{array}{l}\text { Node de- } \\
\text { gree }\end{array}$ & Chance \\
\hline 1 & More & Dense & Very high \\
\hline 2 & More & Fair & High \\
\hline 3 & More & Sparse & Quite high \\
\hline 4 & Average & Dense & High average \\
\hline 5 & Average & Fair & Average \\
\hline 6 & Average & Sparse & Low average \\
\hline 7 & Low & Dense & Quite low \\
\hline 8 & Low & Fair & Low \\
\hline 9 & Low & Sparse & Very low \\
\hline
\end{tabular}

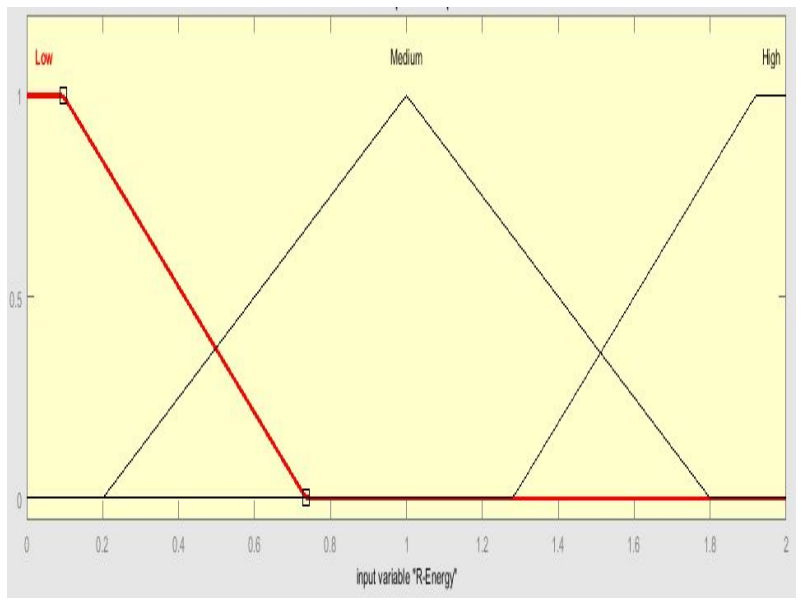

Fig. 2: Fuzzy input for residual energy

$\mu_{A 1}(x)=\left\{\begin{array}{cc}0 & x \leq a \\ \frac{x-a}{b-a} & a \leq x \leq b \\ \frac{c-x}{c-b} & b \leq x \leq c \\ 0 & c \leq x\end{array}\right.$

$\mu_{A 1}(x)=\left\{\begin{array}{cc}0 & x \leq a_{1} \\ \frac{x-a_{1}}{b_{1}-a_{1}} & a_{1} \leq x \leq b_{1} \\ 1 & b_{1} \leq x \leq c_{1} \\ \frac{d_{1}-x}{d_{1}-c_{1}} & c_{1} \leq x \leq d_{1} \\ 0 & d_{1} \leq x\end{array}\right.$

After the computation of chance value, all the nodes in the network will broadcast a $C H$-NOMINEE message containing node $I D$ and its chance value, in their radio range $R$. The higher chance value nodes will elect themselves as $\mathrm{CH}$ and transmit $\mathrm{CH}$-WIN message within its range. The various messages used in $\mathrm{CH}$ election and cluster formation are presented in Table 4.

\subsubsection{Cluster Formation}

During the selection of the $\mathrm{CH}$, a node may receive more than one $C H$-WIN message. If a node receives multiple

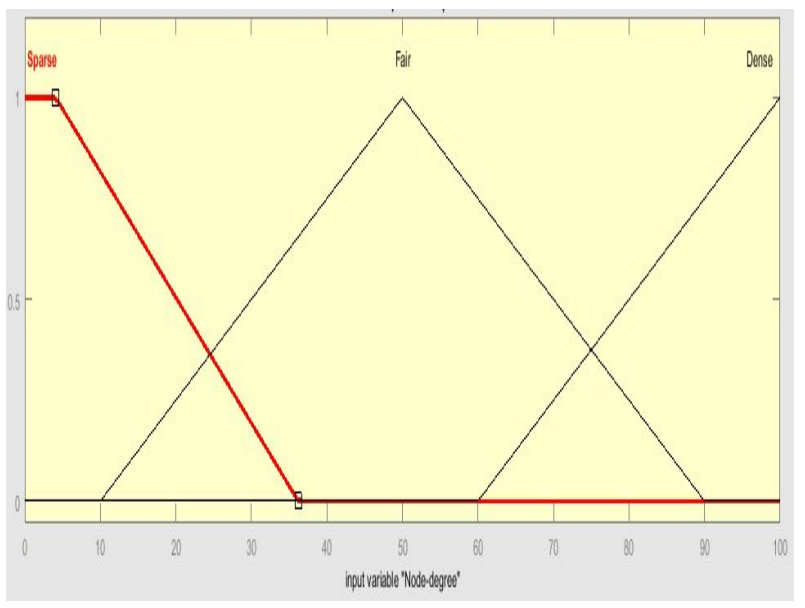

Fig. 3: Fuzzy input for node degree

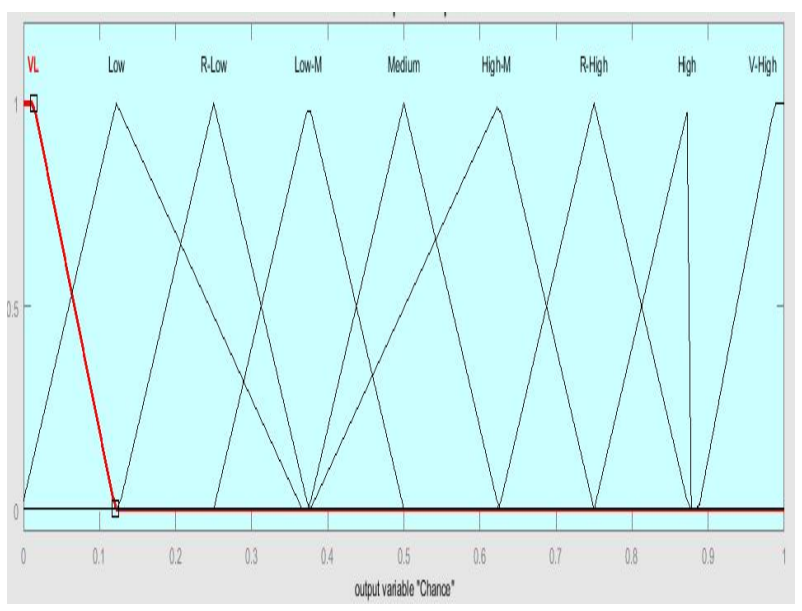

Fig. 4: Fuzzy set for output variable Chance

1. If (R-Energy is Low) and (Node-degree is Sparse) then (Chance is VL) (1)
2. If (R-Energy is Low) and (Node-degree is Fair) then (Chance is Low) (1)
3. If (R-Energy is Low) and (Node-degree is Dense) then (Chance is R-Low) (1)
4. If (R-Energy is Medium) and (Node-degree is Sparse) then (Chance is Low-Mi) (1)
5. If (R-Energy is Medium) and (Node-degree is Fair) then (Chance is Hedium) (1)
6. If (R-Energy is Medium) and (Node-degree is Dense) then (Chance is High-M) (1)
7. If (R-Energy is High) and (Node-degree is Sparse) then (Chance is R-High) (1)
8. If (R-Energy is High) and (Node-degree is Fair) then (Chance is High) (1)
9. If (R-Energy is High) and (Node-degree is Dense) then (Chance is V-High) (1)

Fig. 5: Fuzzy if-then rules 
Table 4: Control Messages used in this scheme

\begin{tabular}{|l|l|}
\hline Message & Functions \\
\hline NODE MSG & $\begin{array}{l}\text { Message containing node ID and } \\
\text { residual energy, broadcast it in their } \\
\text { range } R \text { to gather neighbours infor- } \\
\text { mations }\end{array}$ \\
\hline$C H-N O M I N E E$ & $\begin{array}{l}\text { Broadcast in their communication } \\
\text { range } R\end{array}$ \\
\hline$C H-W I N$ & $\begin{array}{l}\text { Broadcast within its communicated } \\
\text { radius } R \text { to announce himself as a } \\
\mathrm{CH}\end{array}$ \\
\hline$C M-J O I N$ & $\begin{array}{l}\text { normal nodes send it to the nearest } \\
\mathrm{CH} \text { to join it }\end{array}$ \\
\hline$C M-A C C E P T$ & $\begin{array}{l}\mathrm{CH} \text { reply after receiving a CM-JOIN } \\
\text { message }\end{array}$ \\
\hline
\end{tabular}

$C H$-WIN messages, it will associate with minimum distance $\mathrm{CH}$ by sending $C M-J O I N$ message. When $\mathrm{CH}$ receives $C M-J O I N$ message, it will reply with $C M-A C C E P T$ message to it and allow it to join with the $\mathrm{CH}$. In this way, all the nodes in the network are associated with its $\mathrm{CH}$. Associated nodes with its $\mathrm{CH}$ forms a cluster. After the cluster formation, some uncovered sensor nodes remain because they do not join any $\mathrm{CH}$. The uncovered nodes are not covered by the communication range of any selected CHs. Therefore, the uncovered nodes follow a different approach to form the cluster. In this approach, the uncovered nodes calculate their total average distance with each of the uncovered nodes within its range or neighbor nodes. The total average distance for a node is the sum of all the distances between the node to another uncovered neighbor nodes divided by the total number of uncovered neighbor nodes. Now, the node which has the lowest total average distance will declare itself as $\mathrm{CH}$. If there are again some uncovered single nodes present and all the neighbor nodes are covered, then they will declare themselves as CHs. Figure 6 shows the cluster formation scenario, where solid dots are CHs.

Algorithm 1 depicts the whole process of $\mathrm{CH}$ selection and cluster formation. In this algorithm, the pseudocode from line number 3 to 23 represent the selection of final $\mathrm{CH}$ using FIS. From line numbers 24 to 31 are for the uncovered nodes; these are not associated with any $\mathrm{CH}$. Figure 6 illustrated the final cluster formation of 1000 nodes in $500 \mathrm{X} 500 \mathrm{~m}^{2}$ area.

\subsection{Path Planning Phase}

Since our proposed scheme, the $\mathrm{CH}$ election is distributed in nature so that only sensor nodes will take part in the $\mathrm{CH}$ election, and the mobile sink is unaware of $\mathrm{CHs}$. So, to make the mobile sink identify the position of the

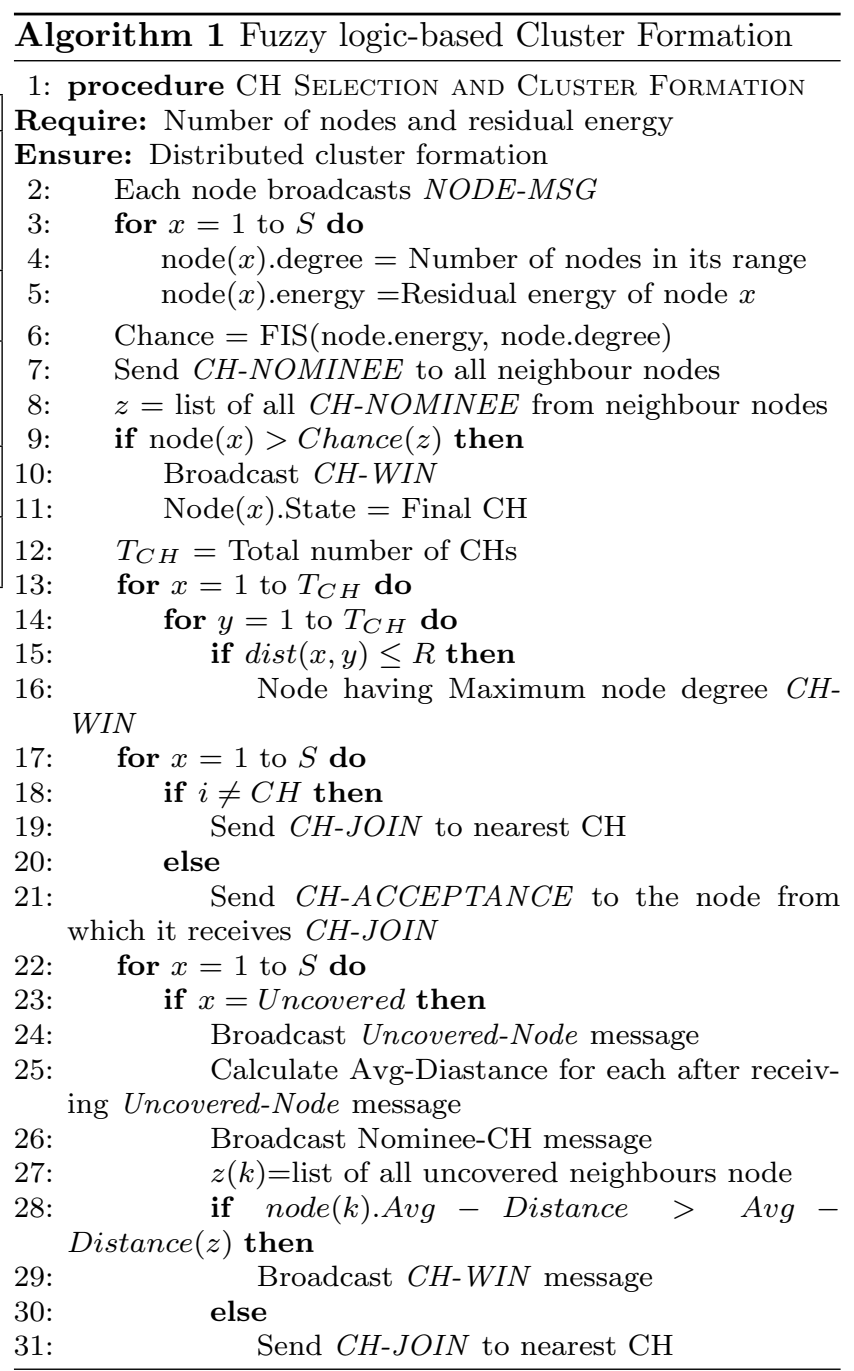

CHs, it will have to travel in two circular paths of radius $80 \mathrm{~m}$ and $240 \mathrm{~m}$ before data collection trajectory for 500X500 $\mathrm{m}^{2}$ area.

When the mobile sink travels in the circular path of radius $80 \mathrm{~m}$, it will get to know all the CHs within 160 $m$ radius since we have assumed that the communication range of sensor nodes is $80 \mathrm{~m}$. Similarly, when the mobile sink moves in the circular path of radius 240 $m$, it will know the locations of all the CHs within a radius of $320 \mathrm{~m}$ except the $\mathrm{CHs}$ within a radius $160 \mathrm{~m}$ as shown in 7 . The CHs transmit their control information like location, remaining energy, node degree, etc. to the mobile sink when both are in the communication range. Now, the mobile sink knows the locations of all the CHs. The mobile sink follows a similar process in case of $1000 \mathrm{X} 1000 \mathrm{~m}^{2}$, but the circular path increases. After knowing the locations of all the CHs, the mobile sink creates the Minimum Spanning Tree (MST) of all the $\mathrm{CHs}$ using the distance between the selected CHs. The MST is started from the centroid of the network, 


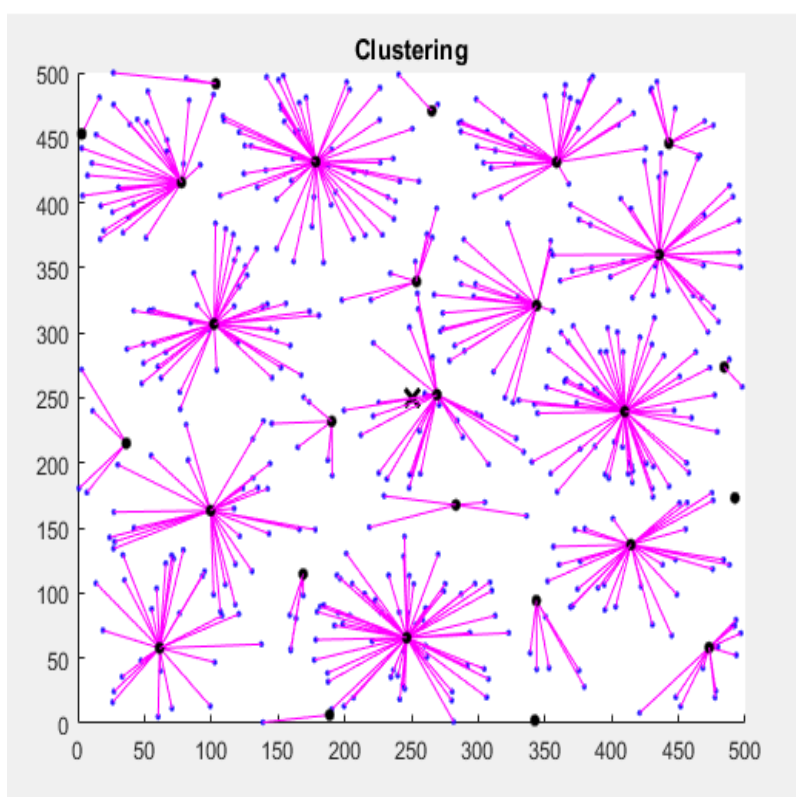

Fig. 6: Cluster formation scenario of 1000 nodes

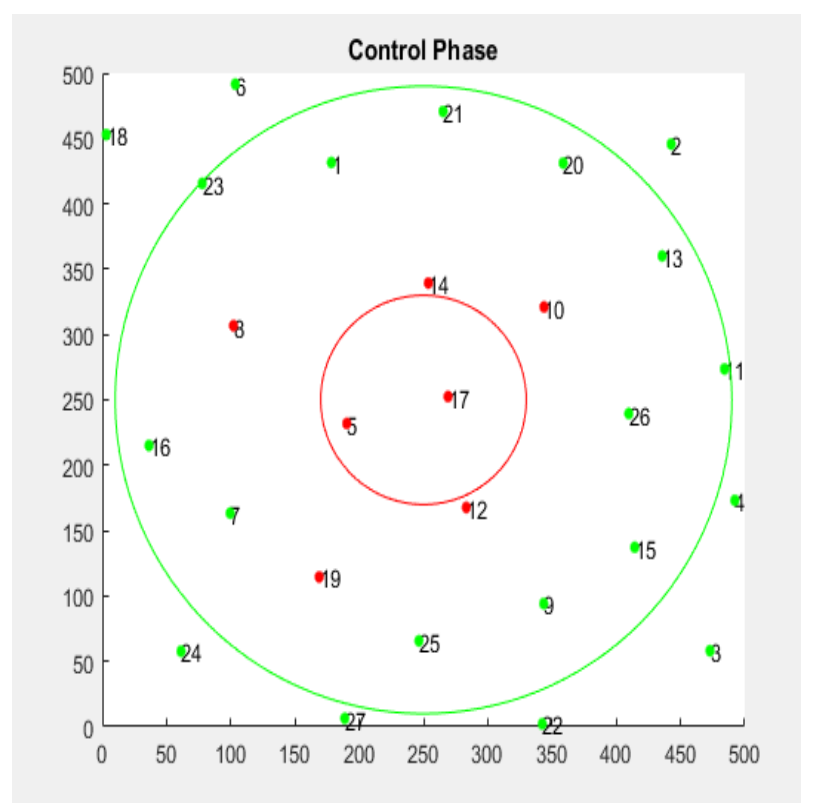

Fig. 7: Circular movement of mobile sink for information collection

and if the two or more $\mathrm{CHs}$ distance from the mobile sink is the same, then the lowest node $I D$ node will get preference. The MST is created to know the cost to reach each $\mathrm{CH}$ in the network. Here, the cost refers to the distance between $\mathrm{CHs}$, which can be shown in Figure 8 .

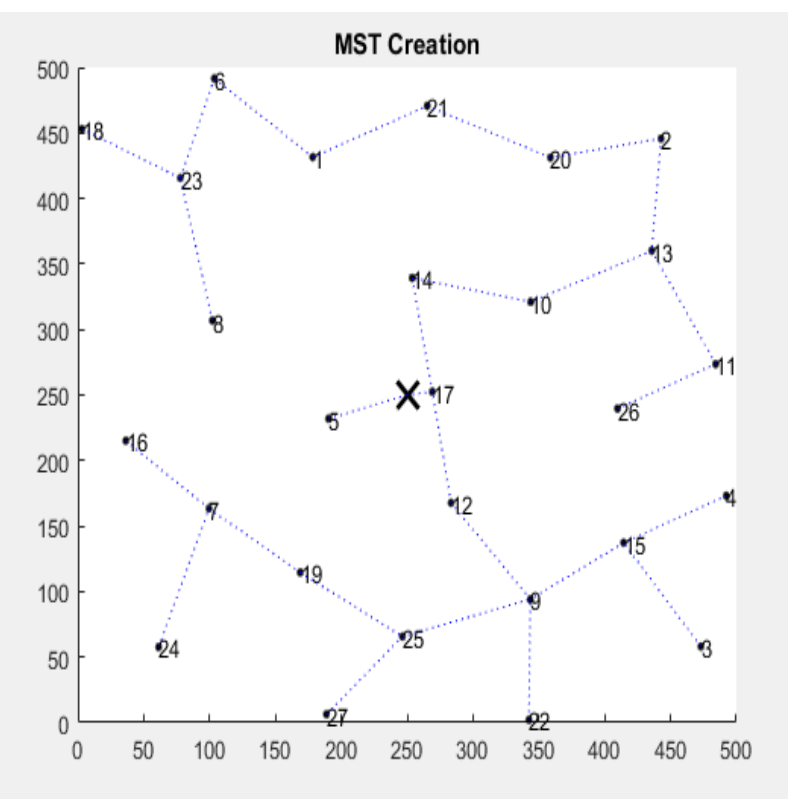

Fig. 8: MST creation for cost calculation

3.4 Technique for Order Preference by Similarity to Ideal Solution (TOPSIS)

At the end of two circular movements, the mobile sink has all the information of each $\mathrm{CH}$. Based on this information, it derives the rank from visiting each $\mathrm{CH}$ by using the TOPSIS method. The TOPSIS is the most famous and widely used multi-criteria decision method developed by Yoon and Hwang in 1981, and later on, many researchers have added extra features and proposed newer version Tzeng and Huang (2011). In the new version, two reference parameters positive ideal solution (PIS) and negative ideal solution (NIS) are introduced. The PIS ideal solution gives the ideal value for all selected criteria, and the NIS produces the worst value for all selected criteria. Generally, the PIS maximizes the benefits and minimizes the total cost, whereas the (NIS) does the reverse process of the PIS. The TOPSIS method uses Euclidean distances to find the relationship among the selected alternatives and the two reference parameters to rank the alternative. The firstrank must be the closest one from the PIS and far away from the NIS.

In the TOPSIS method, assigning the proper weights for different parameters during the formation of the weighted normalized matrix is a big challenge. To handle this with the best solution, the Analytical network process (ANP) Saaty (2013) is applied, which is a widely used tool for parameter selection in many areas. It is a multi-criteria decision tool developed by Saaty to scale parameters and alternatives. A pairwise comparison is 
made to find the relative significance over different parameters and alternatives. To check whether comparisons are reliable or not, it compares with a consistency ratio. If its value is less than 0.1 , then that comparison is consistent, otherwise, repeat the comparison. The weights $\left(w_{1}, w_{2}, w_{3}\right)$ are allotted to each parameter according to its priority which is calculated by ANP. The sum of all weights assigned to each parameter is always 1 as shown in Equation 6.

$w_{1}+w_{2}+w_{3}=1$

In this work, the TOPSIS method is applied to sort the data collection points based on three attributes. The complete processes of the TOPSIS method to short the $\mathrm{CHs}$ for visiting them in a typical fashion are as follows:

1. Initially, the formulation of evaluation matrix using $m$ alternatives and $n$ criteria is obtained where $X_{i j}$ is the intersection of $m$ and $n$, here $m$ alternatives are $\mathrm{CHs}$, and $n$ criteria are energy, node density, and distance which finally gives the $\left(X_{i j}\right)_{m * n}$ matrix as shown in Equation 7

$$
\left(X_{i j}\right)_{m * n}=\left[\begin{array}{cccc}
X_{11} & X_{12} & \ldots \ldots & X_{1 n} \\
X_{21} & X_{22} & \ldots \ldots & X_{2 n} \\
\cdot & \cdot & \cdot & \cdot \\
\cdot & \cdot & \cdot & \cdot \\
\cdot & \cdot & \cdot & \cdot \\
X_{m 1} & X_{m 2} & \ldots . & X_{m n}
\end{array}\right]
$$

2. The matrix $\left(X_{i j}\right)_{m * n}$ is normalized using Equation 9 to get the new matrix $R$

$$
r_{(i j)}=\frac{X_{i j}}{\sum_{k=l}^{m} x_{k j}^{2}}, l=1,2,3, \ldots \ldots, m
$$

The normalised decision matrix represented written as Equation 9.

$$
R=\left[\begin{array}{cccc}
R_{11} & R_{12} & \ldots \ldots & R_{1 n} \\
R_{21} & R_{22} & \ldots \ldots & R_{2 n} \\
\cdot & \cdot & \cdot & \cdot \\
\cdot & \cdot & \cdot & \cdot \\
\cdot & \cdot & \cdot & \cdot \\
R_{m 1} & R_{m 2} & \ldots \ldots & R_{m n}
\end{array}\right]
$$

3. Calculate the weighted normalized decision matrix $P$ by multiplying the respective weight with each column of matrix $R$.

$T_{i j}=r_{i j} . w_{j}, i=1,2,3, \ldots . ., m$ and $j=1,2,3, \ldots ., n$ where $\sum_{j=1}^{n} w_{j}=1$ Then, the weighted normalised matrix will be shown in Equation 10.

$$
P=\left[\begin{array}{cccc}
W_{11} & W_{12} & \ldots \ldots & W_{1 n} \\
W_{21} & W_{22} & \ldots \ldots & W_{2 n} \\
\cdot & \cdot & \cdot & \cdot \\
\cdot & \cdot & \cdot & \cdot \\
\cdot & \cdot & \cdot & \cdot \\
W_{m 1} & W_{m 2} & \ldots . . & W_{m n}
\end{array}\right]
$$

4. Determine the optimal data collection point order in the form of PIS $\left(S^{+}\right)$and worst in the form of NIS $\left(S^{-}\right)$solutions.

$S^{+}=\left[\max _{i}\left(V_{i j} \mid j \in J\right), \min _{i}\left(V_{i j} \mid j \in J\right)\right.$ where

$i=1,2,3, \ldots ., m]$

$=\left[v_{1}^{+}, v_{2}^{+}, \ldots \ldots v_{n}^{+}\right]$

$S^{-}=\left[\min _{i}\left(V_{i j} \mid j \in J\right), \max _{i}\left(V_{i j} \mid j \in J\right)\right.$ where

$i=1,2,3, \ldots ., m]$

$=\left[v_{1}^{-}, v_{2}^{-}, \ldots \ldots v_{n}^{-}\right]$

Where benefit criteria is $j=1,2,3, \ldots . n$

Where cost criteria is $J=1,2,3, \ldots n$

5. Find the separation measure for both the solutions. PIS separation

$S_{i}^{+}=\sqrt{\sum_{j=1}^{n}\left(v_{i j}-v_{j}^{+}\right)}, \quad i=1,2,3, \ldots \ldots . ., m$

NIS separation

$S_{i}^{-}=\sqrt{\sum_{j=1}^{n}\left(v_{i j}-v_{j}^{-}\right)} \quad i=1,2,3, \ldots \ldots ., m$

6. Derive the relative closeness of PIS and NIS separation.

$c_{i}^{+}=\frac{S_{i}^{-}}{S_{i}^{+}+S_{i}^{-}}, \quad 0<c_{i}^{+}<1, \quad i=1,2,3, \ldots \ldots, m$

7. Rank the preference order. A set of alternatives can now be preference ranked according to descending order of $c_{i}^{+}$.

- CH.Energy: Residual Energy of each CH.

- CH.Density: Number of cluster members in the respective $\mathrm{CH}$.

- $E C_{C H}$ : Distance of each $\mathrm{CH}$ from the current position of mobile sink.

- W: Weight vector which stores the weight given for each attribute.

- $A(i, j)$ : Normalized form for each $j$ attribute and $i$ $\mathrm{CH}$.

- $P$ : Weighted matrix which is formed by multiplying the normalized matrix $A$ to the weight vector $W$.

- PIS: It stores the positive ideal solution for each $\mathrm{CH}$.

- NIS: It stores the negative ideal solution for each $\mathrm{CH}$.

- SMP: It is the separation measure of PIS from normalized form for each $\mathrm{CH}$.

- SMN: It is the separation measure of NIS from normalized form for each $\mathrm{CH}$.

- $C$ : Relative closeness to an ideal solution.

$-R_{\text {order }}$ : It is the sorted list of $\mathrm{CHs}$ by its ranking.

$-N_{C H}$ : Total number of CHs in the network. 


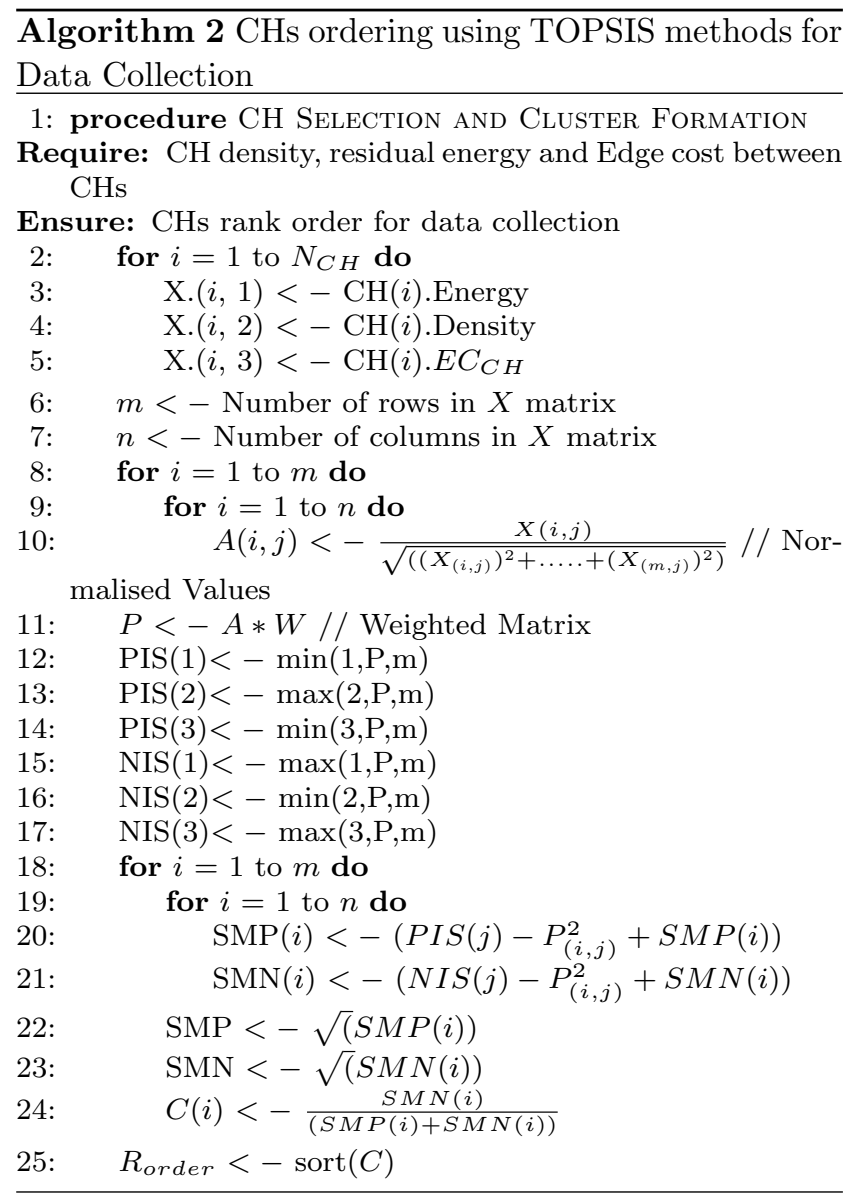

\subsection{Data Collection Phase}

After the path planning phase, the mobile sink has information on residual energy, node degree, and the edge cost of each CH. It will be difficult for the mobile sink to decide which $\mathrm{CH}$ to visit in what order. So, in order to give ranking to them, a multi-criteria decision making TOPSIS method is used, which selects the best alternative among a set of multiple alternatives against multiple criteria. There are several methods for this problem, but the TOPSIS method is used due to its simplicity and better performance.

The residual energy (RE), node degree (ND), and edge cost $\left(E C_{C H}\right)$ of each $\mathrm{CH}$ are applied as criteria to rank them. The residual energy and edge cost are cost attributes, and node degree is the benefit attribute. In the TOPSIS, to find the best alternatives cost attributes are minimized, and the benefit attributes are maximized. The final path of the mobile sink can be seen in Figure 9. The procedure for path planning and data collection phase is given in Algorithm 2. The several terminologies used in this algorithm are mentioned below. The mobile sink moves according to the sorted $\mathrm{CHs}$ based on the multiple-criteria using the TOPSIS method. The Data

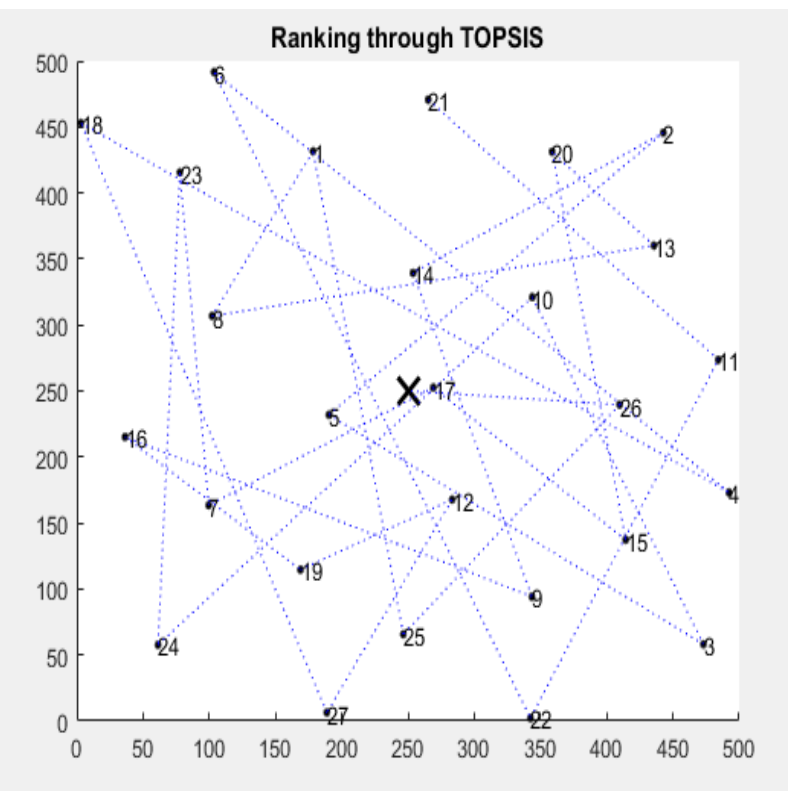

Fig. 9: Data collection trajectory path for mobile sink

Table 5: Simulation parameters

\begin{tabular}{|l|l|}
\hline Parameters & Values \\
\hline Deployment area & $500-1000 \mathrm{~m}^{2}$ \\
\hline Transmission range & $80 \mathrm{~m}$ \\
\hline Node density & 500 to 2000 \\
\hline Initial energy & 2 Joules \\
\hline Data packet size & $1000 \mathrm{bits}$ \\
\hline Control packet size & $120 \mathrm{bits}$ \\
\hline $\mathrm{E}_{\text {elect }}$ & $50 \mathrm{~nJ} / \mathrm{bit}$ \\
\hline $\mathrm{e}_{f s}$ & $10 \mathrm{pJ} / \mathrm{bit} / \mathrm{m}^{2}$ \\
\hline $\mathrm{e}_{m p}$ & $0.0013 \mathrm{pJ} / \mathrm{bit} / \mathrm{m}^{4}$ \\
\hline Antenna type & Omnidirectional \\
\hline MAC protocol & $\mathrm{IEEE} 802.15 .4$ \\
\hline Traffic type & $\mathrm{CBR}(\mathrm{Constant}$ Bit Rate $)$ \\
\hline Mobile sink speed & $5 \mathrm{~m} / \mathrm{s}$ \\
\hline
\end{tabular}

collection trajectory path is illustrated in Figure 9, and the ranks are shown for each $\mathrm{CH}$. Now, the mobile sink will move according to the ranked $\mathrm{CHs}$ to collect the data starting from the $\mathrm{CH}$ having rank 1 . We have considered the speed of the mobile sink to be $5 \mathrm{~m} / \mathrm{s}$. The mobile sink will go near the $\mathrm{CH}$ and send a beacon message to collect the data from each $\mathrm{CH}$. The $\mathrm{CH}$ will acknowledge the beacon message and then sends their collected data.

\section{Simulation results and Performance analysis}

In order to check our scheme's performance in a large sensor network, we have simulated it in two different sizes of networks. The size of both networks are 500X500 $\mathrm{m}^{2}$ and 1000X1000 $\mathrm{m}^{2}$ with 1000 and 2000 sensor nodes, 
respectively. In both scenarios, nodes are placed in random mode, and after placement, all nodes are static. The proposed algorithm is compared with two existing protocols like Kaswan et al (2017) and Almi'ani et al (2010). Both protocols are similar to our scheme in terms of mobile sink-based data collection and sojourn points. In the RP Kaswan et al (2017) based protocol, RPs are calculated based on node density, and TSP decides routes of the mobile sink. Whereas, the CP-based scheme Almi'ani et al (2010), has designed a predefined path based on a grid cluster's cache points (CP). In the plotted graph, we have used MC (multicriteria-based), RP (rendezvous point-based), and CP (cache point-based) notations for better convenience. With the same set-up and 20 different simulation scenarios, average results have been considered for plotting the graphs.

The MATLAB R2017a tool is used for the simulation of our scheme. Table 5 demonstrates all the basic simulation parameters we have applied in this work.

\subsection{Performance analysis}

Average residual energy, first node dead (FND), half node dead (HND), last node dead (LND), number of collection points, node density, and number of rounds, etc. are the performance metrics used for the comparison. In our scheme, the data collection point is the number of $\mathrm{CHs}$, and due to distributed clustering, whenever new clusters will form the numbers of data collection points are changed. Experimental analysis of the proposed work for both the scenarios on different parameters are mentioned in subsequence sub-sections.

\subsection{Results analysis in FND, HND, and LND}

In the beginning, after random deployment, every node $\left(N_{i}\right)$ finds its coordinates $\left(A_{i}, B_{i}\right)$ by way of using an efficient localization scheme Singh et al (2017a). Afterward, each node $N_{i}$ broadcasts a control packet with its details such as node $I D$, present-day strength and location coordinates. Each node maintains a neighbor table, which helps to calculate the node degree (total number of nodes in the range of a precise node) of that node. Suppose, a node $N_{i}$ receives a control packet from $N_{j}$, then the updated neighbour table of $N_{i}$ will be $\operatorname{Nbr}\left(N_{i}\right)=\left[\operatorname{Nbr}\left(N_{i}\right) \cup N_{j}\right]$. After that, all nodes will be aware of the neighbor's statistics like their distance, power, etc. This information will help in modeling the FIS for $\mathrm{CH}$ decision and cluster formation. The set-up section, more often than not, consists of $\mathrm{CH}$ selection and cluster formation. In this phase, clusters are shaped

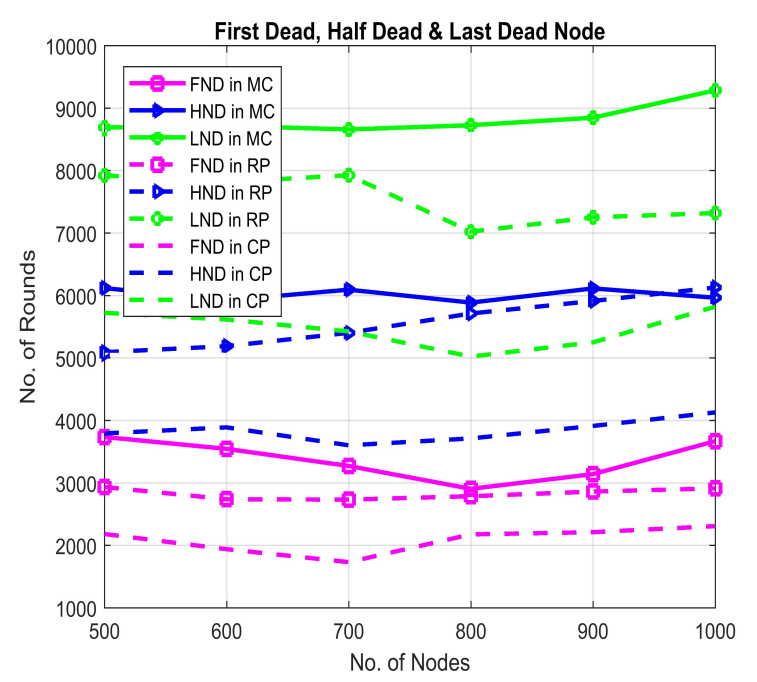

Fig. 10: FND, HND and LND with respect to round number in first scenario.

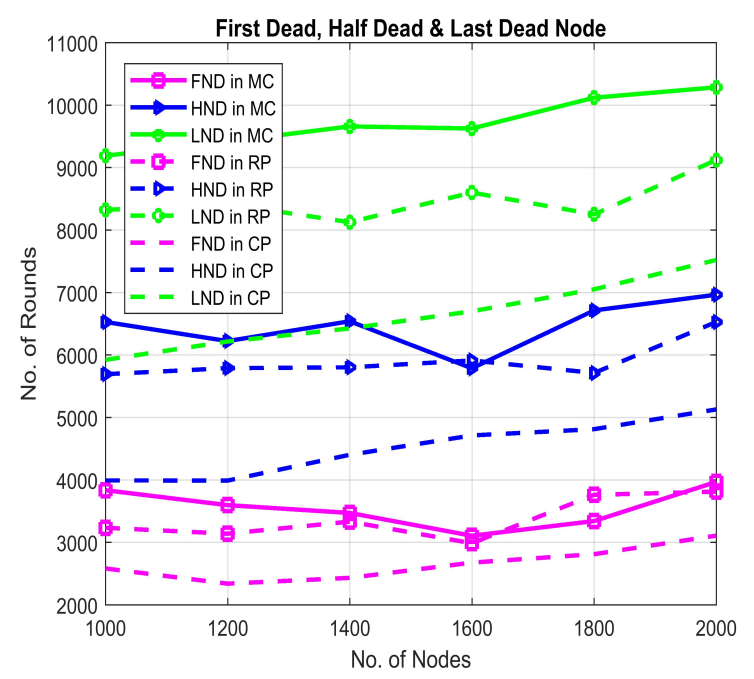

Fig. 11: FND, HND and LND with respect to round number in second scenario.

with the help of FIS, and it is completely allotted in nature because of $\mathrm{CH}$ selection and cluster formation function based on local data only.

\subsection{Network Lifetime}

It is an essential metric for evaluating network performance. The network lifetime depends on the number of rounds a network operates. Figures 12 and 13 illustrate the performance comparison with the number of rounds and average residual energy.

The average remaining energy behaviors on the network are given in Figure 12. Two protocols RP and CP are 


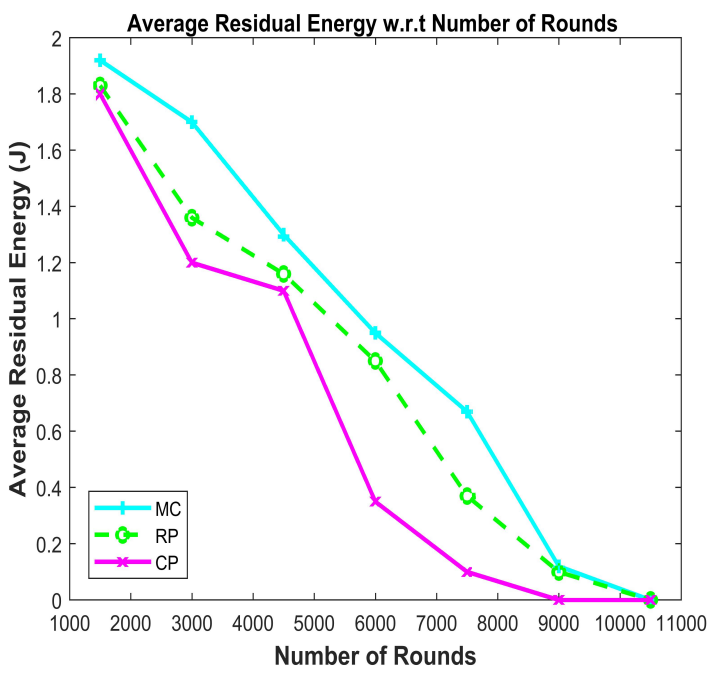

Fig. 12: Average residual energy vs number of rounds in first scenario.

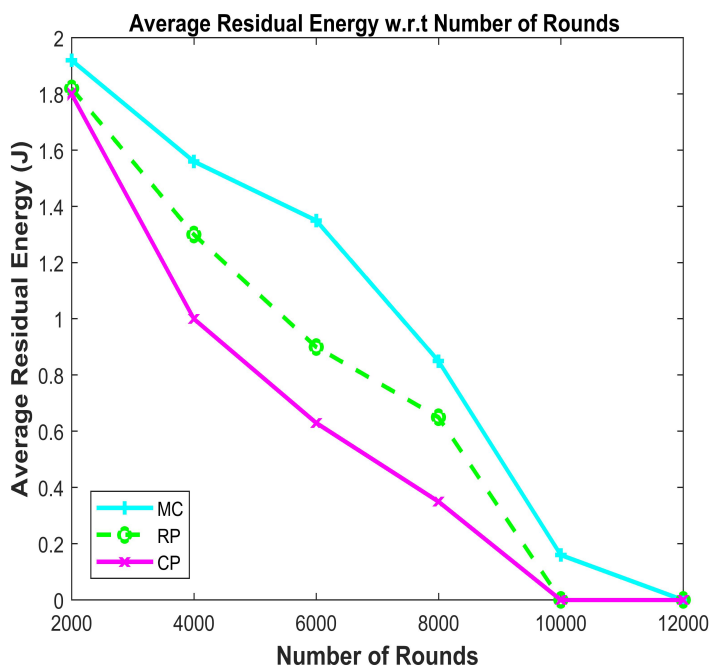

Fig. 13: Average residual energy vs number of rounds in second scenario.

used to compare with our scheme in the first scenarios. It can be seen from the figure that this scheme lasts more than 10000 rounds, but in the same set-up, the RP and CP depleted their total energy under 9000 and 9500 rounds, respectively. Due to the smooth energy depletion, the hotspot problem does not occur, and as a result, it sustains up to 10000 . Our scheme is superior to other schemes because it uses optimal $\mathrm{CH}$ selection and path planning. In MC, sensor nodes drain their energy gradually at a constant rate; it results in a low death rate of sensor nodes.

The simulation results plotted in Figure 13 illustrates the network lifetime of our scheme in a large sensor

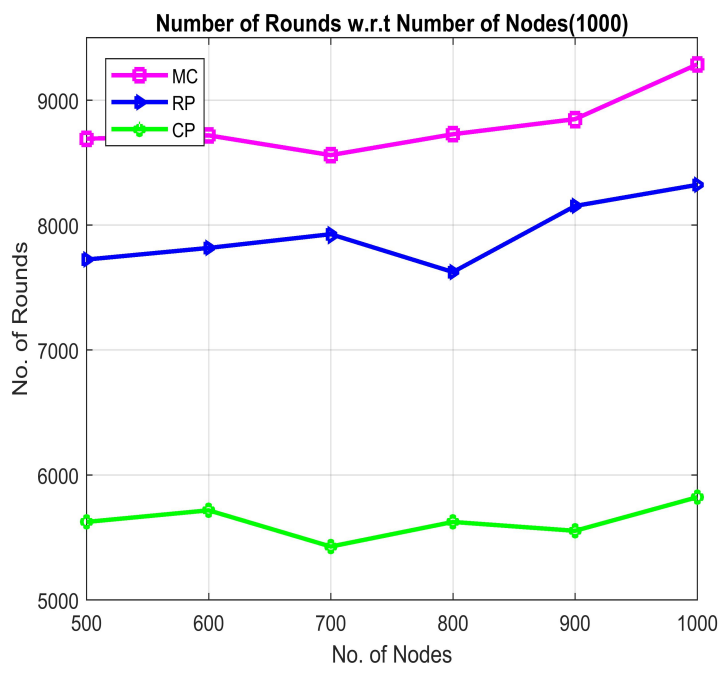

Fig. 14: Number of nodes vs number of rounds in first scenario.

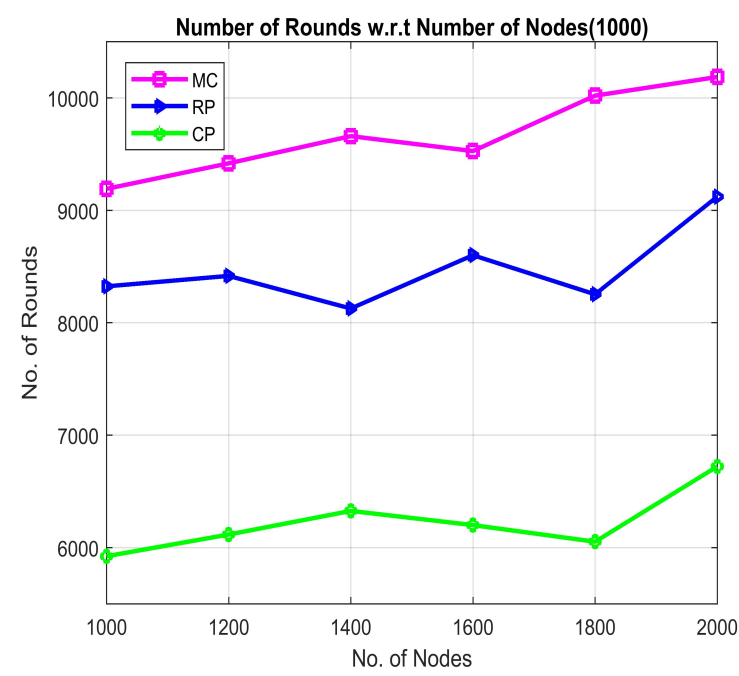

Fig. 15: Number of nodes vs number of rounds in second scenario.

network with a higher number of nodes. As we can see from the figure, in the second scenario, our scheme also performed well than RP and CP. Observation-based on the figure shows MC attains higher life with low energy depletion per round as compare to other schemes. Our scheme survives around 12000 rounds, as we can see that in both the scenarios, only approx 2000 rounds difference is in their lifetime. This is due to the large sensor network with higher nodes with extra overheads and more data to carry. However, our scheme also achieves a better improvement in a large sensor network, but $\mathrm{RP}$ and $\mathrm{CP}$ fail to achieve a higher life in a large sensor network. 


\subsection{Effect of node density}

Similarly, we compare it in a different number of nodes with the number of rounds. Figures 14, and 15 illustrated the performance evaluations in two different scenarios with two existing protocols.

Figure 14 illustrates the comparison between different node densities (500 to 1000) with round numbers. The proposed scheme maximizes the network lifetime by surviving more number of rounds and can transmit more packets to the mobile sink. Our scheme outperforms the RP method by $11 \%$ and the CP by $42 \%$. This is because the MC scheme selects a more stable node as a $\mathrm{CH}$ and collects data with a multi-criteria decision based (TOPSIS) method. None of the schemes has considered all of these parameters together for path planning. $\mathrm{RP}$ is based on node density and multi-hop path only, whereas node energy and MC decision technique are not considered. While CP is based on a grid-based cluster and after clustering, it selects some cache points based on the communication range, and also it has a predefined path for the mobile sink. Here, we have considered three parameters for the multi-criteria decision, and for $\mathrm{CH}$ selection, we use a fuzzy controller, which results in more rounds.

Figure 15 shows the total round numbers protocols sustain in different node densities in the large sensor network. The proposed scheme also performs better in the second scenario as compared to RP and CP. In the large area, it has $8 \%$ and $35 \%$ more lifetime as compared to RP and CP methods, respectively. The reason is efficient clustering and less number of data collection points. The other causes for better performance have already discussed in the first scenario. Therefore, we can efficiently use our scheme in large sensor networks.

\subsection{Effect of node density on data collection points}

In mobile sink based WSN, the information collection points are an essential aspect of achieving higher performance. If the collection points are much less, then delay will be minimal, and the number of communications is also minimized, whereas in a large number of collection points, the delay will be extra and extra power is consumed due to more communications overheads. Figures 16 and 17 depict the contrast of MC and existing protocols based on the number of information collection points for different node density ranging from 500 to 1000 for the first scenario and 1000 to 2000 nodes for the second scenario respectively. The information collection points are differ based on the techniques used for

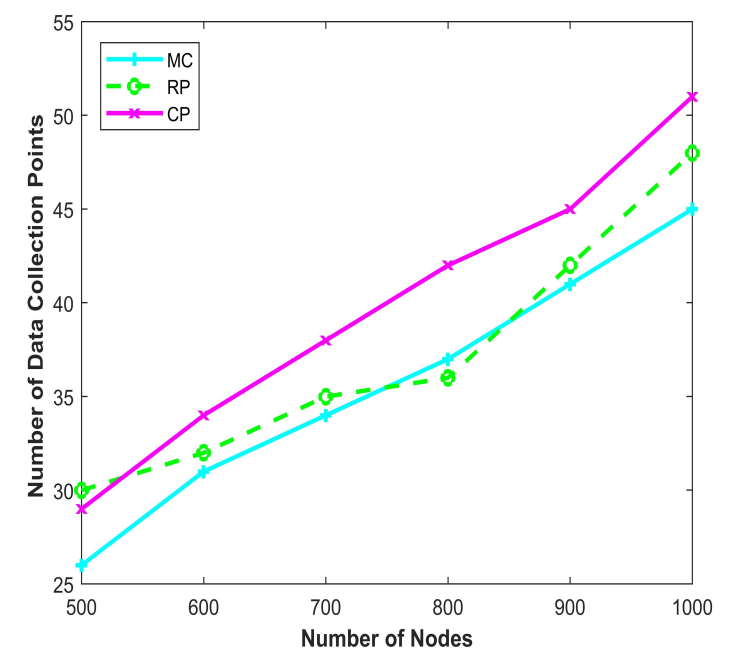

Fig. 16: Number of Data collection points vs number of nodes in first scenario.

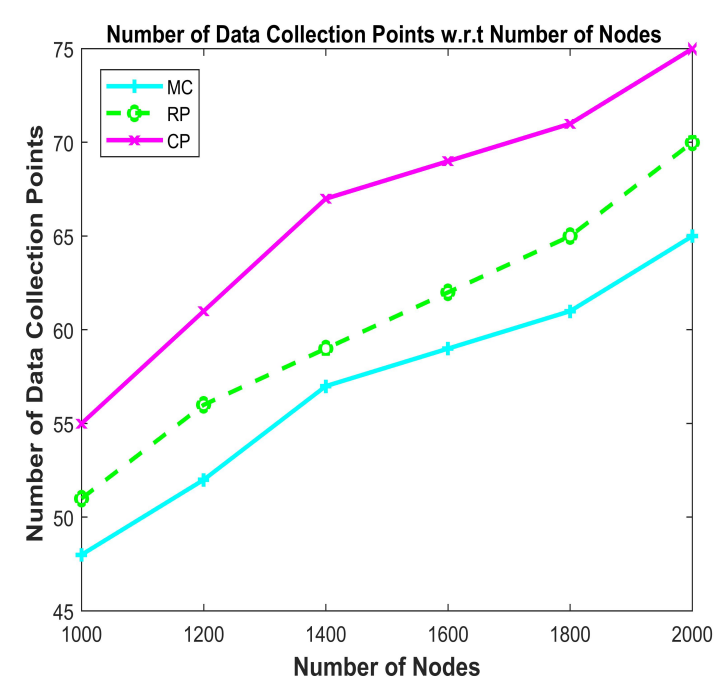

Fig. 17: Number of Data collection points vs number of nodes in second scenario.

discovering collection points. In our scheme, the number of $\mathrm{CHs}$ are counted as the information collection points, whereas in RP based techniques, some points are selected based on few parameters like node density, distance from the mobile sink, etc.

Figure 16 shows the complete data collection points shaped for different node densities in the first scenario. As can be seen from the figure, our scheme has fewer data collection points than RP and CP, and this is due to distributed fuzzy clustering with more than one parameter. This is the primary motive for our protocol to have higher life due to much less data collection points, where communication overheads get reduced. 


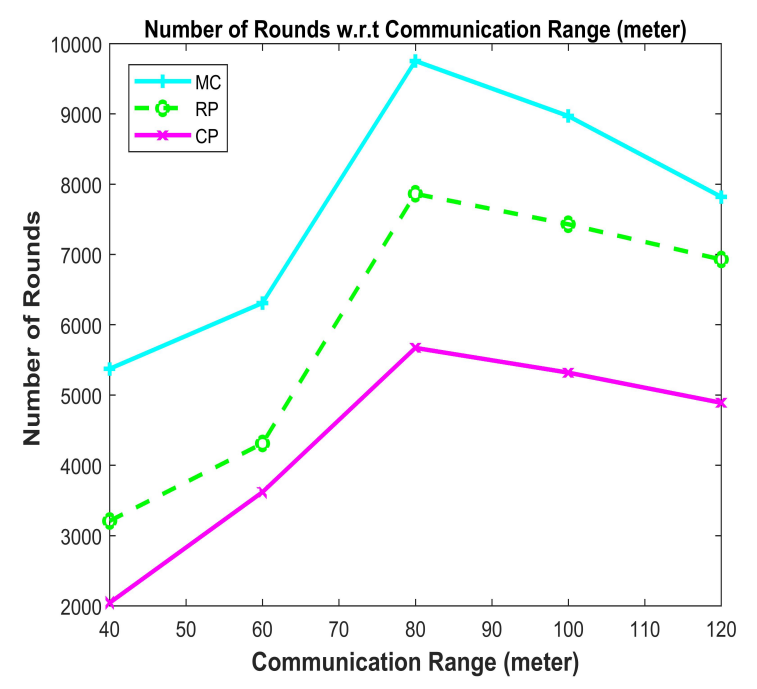

Fig. 18: Communication range vs Number of rounds in first scenario.

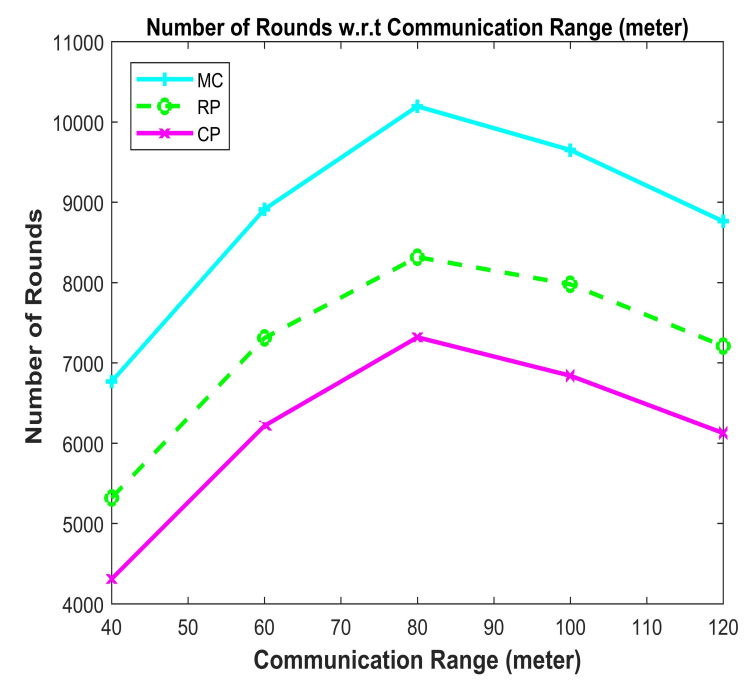

Fig. 19: Communication range vs Number of rounds in first scenario.

Figure 17 displays the data collection points formed for distinctive node densities in a large sensor network. Here, we can also see that a lower number of data collection points are formed in our scheme compared to the other two schemes. This minimizes the power consumption in a large sensor network and prolongs the lifetime. In RP and CP greater range of data collection points are created, which will increase the extra network overheads. This result proved that our protocol could be efficaciously used in large sensor networks.

\subsection{Communication Range}

Figures 18 and 19 represent the impact of radio ranges on the MC in opposition to RP and CP schemes. We have fixed the value of node range $(R)$ is 80 , and primarily based on this, we constructed the clusters in a distributed nature. Why we have selected 80 only, it will be clear while we see the outcomes in round numbers on the different communication ranges. As we increase the radio range, fewer clusters will be formed and viceversa. Based on the performance graphs, it can be argued that 75 to $90 \mathrm{~m}$ ranges are the best for the overall performance.

Figure 18 denotes the round numbers at distinct communication levels from 40 to $120 \mathrm{~m}$ in the first scenario. It can be considered from the figure that our scheme carried out fantastically in all radio ranges as compared to RP and CP. In higher radio range, all protocols showing a shorter lifetime. More clusters generate in the low radio range, which directs to new transmissions and data collection points. In higher radio range, the intra-cluster routing cost increases because of large cluster size. Though, in each (lower and higher) transmission ranges, the network lifetime in terms of rounds is terrible; however, between 70 and $100 \mathrm{~m}$, the network lifetime is excellent and most favorable. This is the principal purpose of choosing a default communication range of $80 \mathrm{~m}$.

Figure 19 illustrates the impact of round numbers at various communication levels from 40 to $120 \mathrm{~m}$ in the second scenario. We can see from the figure that the overall performance of all schemes is much less compared to the first scenario. The only difference is in their lifetime; in this situation, the network lifetime is extra as compared to the first scenario. However, the nature of the diagram is similar. All the schemes preserve up to more significant round numbers in-between communication levels of 70 to $90 \mathrm{~m}$. Therefore, the simulation for large sensor networks used the same communication range as in the first scenario.

\section{Conclusions and Future Works}

The paper has discussed a distributed clustering using a fuzzy controller for greater stable cluster formation and a most appropriate route planning mechanism with the help of a multi-criteria-based decision (TOPSIS) scheme. The mobile sink-based data collection solves the hotspot hassle through direct gathering the information from the CHs. The fuzzy rule-based system selected the best-suited node as a $\mathrm{CH}$, which additionally balanced the power consumption. For efficient data 
collection, the TOPSIS approach ranks the CHs in ascending order, so that the mobile sink visits all the $\mathrm{CHs}$ according to this rank order. The center of attention of this work has two fundamental goals: enhancement of network lifetime and scalability, and both have been executed as proven in the results section. Moreover, the experimental analysis shows that the proposed scheme also performs better in large sensor networks, which is an important objective. The multi-criteria decisionbased route planning can be used in different methods, also in WSN like $\mathrm{CH}$ selection, cluster formation, etc. This model's effectivity is measured primarily by node density, network lifetime, number of rounds, transmission range, and number of data collection points. Our scheme achieved greater network lifetime, decrease power consumption, minimum data collection points in small, as well as large sensor networks, compared to other current protocols. Improvements of up to 16-20\% have been achieved in terms of network lifetime with the aid of using a multi-criteria decision-based path planning scheme for cellular sink over existing protocols. In the future, the current work will be prolonged for delay bounded networks with more significant parameters and different network structures.

\section{Acknowledgements}

The authors would like to acknowledge the Ministry of Electronics and Information Technology (MeitY), Government of India, for supporting the financial assistant during research through the "Visvesvaraya Ph.D. Scheme for Electronics and IT."

\section{Compliance with ethical standards}

\section{Conflict of interest}

The authors declare that they have no conflict of interest.

\section{Authorship contributions \\ Sunil Kumar Singh: Conceptualization, Methodol- ogy, Software, Writing- Original draft preparation.}

Prabhat Kumar: Supervision, Validation, WritingReviewing and Editing,

\section{References}

Akyildiz IF, Su W, Sankarasubramaniam Y, Cayirci E (2002) Wireless sensor networks: a survey. Computer networks 38(4):393-422

Almi'ani K, Viglas A, Libman L (2010) Energy-efficient data gathering with tour length-constrained mobile elements in wireless sensor networks. In: IEEE Local Computer Network Conference, pp 582-589

Alrashidi M, Nasri N, Khediri S, Kachouri A (2020) Energy-efficiency clustering and data collection for wireless sensor networks in industry 4.0 pp 1-8

Antoo A, Mohammed AR (2014) Eem-leach: Energy efficient multi-hop leach routing protocol for clustered wsns. In: 2014 International Conference on Control, Instrumentation, Communication and Computational Technologies (ICCICCT), pp 812-818

Bagci H, Yazici A (2013) An energy aware fuzzy approach to unequal clustering in wireless sensor networks. Applied Soft Computing 13(4):1741 - 1749

Baranidharan B, Santhi B (2016) Ducf: Distributed load balancing unequal clustering in wireless sensor networks using fuzzy approach. Applied Soft Computing 40:495-506

Biradar RV, Sawant S, Mudholkar R, Patil V (2011) Multihop routing in self-organizing wireless sensor networks. International Journal of Computer Science Issues (IJCSI) 8(1):155-164

Chang C, Chen S, Chang I, Yu G, Roy DS (2020) Multirate data collection using mobile sink in wireless sensor networks. IEEE Sensors Journal pp 1-1

Chauhan V, Soni S (2019) Mobile sink-based energy efficient cluster head selection strategy for wireless sensor networks. Journal of Ambient Intelligence and Humanized Computing pp 1-14

Chengfa Li, Mao Ye, Guihai Chen, Jie Wu (2005) An energy-efficient unequal clustering mechanism for wireless sensor networks. In: IEEE International Conference on Mobile Adhoc and Sensor Systems Conference, 2005., pp 8 pp.-604

Debasis K, Singh M, Gupta R (2020) An energy saving medium access control protocol for wireless sensor networks. Journal of Ambient Intelligence and $\mathrm{Hu}-$ manized Computing pp 1-14

Fadel E, Gungor V, Nassef L, Akkari N, Maik MA, Almasri S, Akyildiz IF (2015) A survey on wireless sensor networks for smart grid. Computer Communications 71:22-33

Fanian F, Rafsanjani MK (2018) Memetic fuzzy clustering protocol for wireless sensor networks: Shuffled frog leaping algorithm. Applied Soft Computing 71:568-590

Goyal D, Tripathy MR (2012) Routing protocols in wireless sensor networks: a survey. In: 2012 Second International Conference on Advanced Computing \& Communication Technologies, IEEE, pp 474-480

Heinzelman WB, Chandrakasan AP, Balakrishnan H (2002) An application-specific protocol architecture for wireless microsensor networks. IEEE Transactions on wireless communications 1(4):660-670 
Heinzelman WR, Chandrakasan A, Balakrishnan $\mathrm{H}$ (2000) Energy-efficient communication protocol for wireless microsensor networks. In: System sciences, 2000. Proceedings of the 33rd annual Hawaii international conference on, IEEE, pp 01-10

Jain S, Shah RC, Brunette W, Borriello G, Roy S (2006) Exploiting mobility for energy efficient data collection in wireless sensor networks. Mob Netw Appl 11(3):327-339, DOI 10.1007/s11036-006-5186-9

Jannu S, Jana PK (2015) A grid based clustering and routing algorithm for solving hot spot problem in wireless sensor networks. Wireless Networks pp 1-16

Juang P, Oki H, Wang Y, Martonosi M, Peh LS, Rubenstein D (2002) Energy-efficient computing for wildlife tracking: Design tradeoffs and early experiences with zebranet. SIGARCH Comput Archit News 30(5):96107, DOI 10.1145/635506.605408, URL http://doi. acm.org/10.1145/635506.605408

Kaswan A, Nitesh K, Jana PK (2017) Energy efficient path selection for mobile sink and data gathering in wireless sensor networks. AEU - International Journal of Electronics and Communications 73:110 - 118

Kim J, Sajjad Hussain C, Yang W, Kim D, Park M (2008) Produce: A probability-driven unequal clustering mechanism for wireless sensor networks. In: 22nd International Conference on Advanced Information Networking and Applications - Workshops (aina workshops 2008), pp 928-933

Kumar N, Sandeep, Bhutani P, Mishra P (2012) Uleach: A novel routing protocol for heterogeneous wireless sensor networks. In: 2012 International Conference on Communication, Information Computing Technology (ICCICT), pp 1-4

Kumar N, Dash D, Kumar P (2017) Optimal subpath selection for maximum data gathering using mobile sink in wsn. In: Proceedings of the 7th International Conference on Computer and Communication Technology, ACM, New York, NY, USA, ICCCT2017, pp 66-71, DOI 10.1145/3154979.3154997, URL http://doi.acm.org/10.1145/3154979.3154997

Lee S, Choe H, Park B, Song Y, Kim Ck (2011) Luca: An energy-efficient unequal clustering algorithm using location information for wireless sensor networks. Wireless Personal Communications 56(4):715-731, DOI 10.1007/s11277-009-9842-9, URL https:// doi.org/10.1007/s11277-009-9842-9

Liu W, Wang Z, Zhang S, Wang Q (2010) A low power grid-based cluster routing algorithm of wireless sensor networks. In: 2010 International Forum on Information Technology and Applications, vol 1, pp 227229

Logambigai R, Kannan A (2016) Fuzzy logic based unequal clustering for wireless sensor networks. Wire- less Networks 22(3):945-957, URL https://doi. org/10.1007/s11276-015-1013-1

Nabajyoti M, Hari O (2018) Distributed fuzzy approach to unequal clustering and routing algorithm for wireless sensor networks. International Journal of Communication Systems 31(12):1-23, DOI 10.1002/dac.3709, URL https://onlinelibrary. wiley.com/doi/abs/10.1002/dac.3709, e3709 dac.3709, https://onlinelibrary.wiley.com/ doi/pdf/10.1002/dac.3709

Nesamony S, Vairamuthu MK, Orlowska ME (2007) On optimal route of a calibrating mobile sink in a wireless sensor network. In: 2007 Fourth International Conference on Networked Sensing Systems, pp 6164, DOI 10.1109/INSS.2007.4297389

Nitesh K, Kaswan A, Jana PK (2017) Energy density based mobile sink trajectory in wireless sensor networks. Microsystem Technologies

Pal R, Karnwal R, Aarti (2020) Eewc: energy-efficient weighted clustering method based on genetic algorithm for hwsns. Complex \& Intelligent Systemsl pp 1-1, URL doi .org/10.1007/s40747-020-00137-4

Pitke K, Kumar P, Singh SK (2017) A load balancing cross clustering approach in wireless sensor network. In: Proceedings of the 7th International Conference on Computer and Communication Technology, ACM, pp $52-57$

Qiang T, Bingwen W, Zhicheng D (2009) Ms-leach: A routing protocol combining multi-hop transmissions and single-hop transmissions. In: Pacific-Asia Conference on Circuits, Communications and Systems, IEEE, pp 107-110

Rajaram V, Kumaratharan N (2020) Multi-hop optimized routing algorithm and load balanced fuzzy clustering in wireless sensor networks. Journal of Ambient Intelligence and Humanized Computing pp 1-9

Randhawa S, Jain S (2019) Mlbc: Multi-objective load balancing clustering technique in wireless sensor networks. Applied Soft Computing 74:66-89

Rao J, Biswas S (2008) Data harvesting in sensor networks using mobile sinks. IEEE Wireless Communications 15(6):63-70, DOI 10.1109/MWC.2008. 4749749

Saaty TL (2013) Analytic hierarchy process. In: Encyclopedia of operations research and management science, Springer, pp 52-64

Sert SA, Bagci H, Yazici A (2015) Mofca: Multiobjective fuzzy clustering algorithm for wireless sensor networks. Applied Soft Computing 30:151-165

Sha C, Qiu Jm, Lu Ty, Wang Tt, Wang Rc (2017) Virtual region based data gathering method with mobile sink for sensor networks. Wireless Networks 
Shah R, Roy S, Jain S, Brunette W (2003) Data mules: modeling a three-tier architecture for sparse sensor networks. In: Sensor Network Protocols and Applications, 2003. Proceedings of the First IEEE. 2003 IEEE International Workshop on, pp 30-41

Singh SK, Kumar P (2019) A load balancing virtual level routing (lbvlr) using mobile mule for large sensor networks. The Journal of Supercomputing 75(11):74267459 , DOI 10.1007/s11227-019-02952-0, URL https://doi.org/10.1007/s11227-019-02952-0

Singh SK, Kumar P (2020) A comprehensive survey on trajectory schemes for data collection using mobile elements in wsns. Journal of Ambient Intelligence and Humanized Computing 11(3):291-312

Singh SK, Kumar P, Singh JP (2016) An energy efficient odd-even round number based data collection using mules in wsns. In: 2016 International Conference on Wireless Communications, Signal Processing and Networking (WiSPNET), pp 1255-1259

Singh SK, Kumar P, Singh JP (2017a) Localization in wireless sensor networks using soft computing approach. International Journal of Information Security and Privacy (IJISP) 11(3):42-53

Singh SK, Kumar P, Singh JP (2017b) A survey on successors of leach protocol. IEEE Access 5:4298-4328

Singh SK, Kumar P, Singh JP (2018) An energy efficient protocol to mitigate hot spot problem using unequal clustering in wsn. Wireless Personal Communications

Thanigaivelu K, Mururgan K (2012) Grid-based clustering with predefined path mobility for mobile sink data collection to extend network lifeime in wireless sensor network. IETE Technical Review 29(2):133146

Tzeng GH, Huang JJ (2011) Multiple attribute decision making: methods and applications. Chapman and Hall/CRC

Xing G, Wang T, Xie Z, Jia W (2008) Rendezvous planning in wireless sensor networks with mobile elements. IEEE Transactions on Mobile Computing 7(12):1430-1443, DOI 10.1109/TMC.2008.58

Zhao H, Guo S, Wang X, Wang F (2015) Energy efficient topology control algorithm for maximizing network lifetime in wireless sensor networks with mobile sink. Applied Soft Computing 34:539-550 